\title{
The Beck Depression Inventory had low sensitivity and moderate specificity for detecting depression in patients with Alzheimer's disease
} Wagle AC, Ho LW, Wagle SA, et al. Psychometric behaviour of BDI in Alzheimer's disease patients with depression.
Int J Geriatr Psychiatry 2000 Jan;15:63-9.

QUESTION: Is the Beck Depression Inventory (BDI) an appropriate screening tool for depression in patients with Alzheimer's disease (AD)?

\section{Design}

Blinded comparison of the 21 item BDI (revised version) with $I C D-9$ and $I C D-10$ diagnosis of depression.

\section{Setting}

The neuropsychiatric (tertiary referral) clinics at Addenbrooke's Hospital, UK.

\section{Patients}

2 samples were studied: 129 patients with probable AD (AD based on NINCDS-ADRDA criteria, mean age $62 \mathrm{y}$, $64 \%$ men) and 52 with probable $\mathrm{AD}$ plus depression (mean age $62 \mathrm{y}, 52 \%$ men).

Description of test and diagnostic standard The BDI was self administered during a 45 minute computerised testing session. 8 core questionnaires were given during this session one of which was the BDI. A global BDI score was obtained as well as a cognitive score (items 1-13) and a somatic score (items 14-21). The diagnostic standard was ICD-9 and ICD-10.ICD-10 criteria for depression included depressed mood; loss of interest and enjoyment, reduced self esteem, ideas of guilt or unworthiness; bleak and pessimistic view of the future; and ideas of self harm.

\section{Main outcome measures}

Sensitivity, specificity, and positive and negative likelihood ratios (LRs) for each cut off point on the BDI, and area under the receiver operating characteristic (ROC) curve.

\section{Main results}

The table represents sensitivity, specificity, and positive and negative LRs. A BDI score of 12 offered the best compromise. ROC curves for total BDI and cognitive

Sensitivity, specificity, and positive and negative likelihood ratios (LRs) for different Beck Depression Inventory (BDI) cut off

\begin{tabular}{lllll}
$\begin{array}{l}\text { Global } \\
\text { BDl cut } \\
\text { off scores }\end{array}$ & $\begin{array}{l}\text { Sensitivity } \\
(\%)\end{array}$ & $\begin{array}{l}\text { Specificity } \\
(\%)\end{array}$ & $\begin{array}{l}\text { LR for a } \\
\text { positive } \\
\text { test }\end{array}$ & $\begin{array}{l}\text { LR for a } \\
\text { negative } \\
\text { test }\end{array}$ \\
\hline 10 & 65 & 72 & 2.40 & 0.49 \\
\hline 11 & 61 & 75 & 2.56 & 0.52 \\
\hline 12 & 61 & 80 & 3.17 & 0.49 \\
\hline 13 & 53 & 83 & 3.30 & 0.57 \\
\hline 14 & 51 & 85 & 3.52 & 0.58 \\
\hline 15 & 40 & 89 & 3.72 & 0.67 \\
\hline 16 & 36 & 92 & 4.71 & 0.70 \\
\hline 17 & 30 & 92 & 3.96 & 0.76 \\
\hline 18 & 25 & 92 & 3.22 & 0.82 \\
\hline *Diagnostic terms defined in glossary +Numbers calculated from data in article.
\end{tabular}

and somatic item subsets showed low sensitivity and low areas under the curve indices.

\section{Conclusion}

The 21 item revised version of the Beck Depression Inventory had low sensitivity and moderate specificity for detecting depression in patients with Alzheimer's disease.

\section{COMMENTARY}

This study by Wagle $e t$ al found that the BDI was not consistent with $I C D-10$ criteria for diagnosing depression in people with $\mathrm{AD}$. In some ways this is not surprising, as the authors chose the BDI, not because it is the most appropriate scale, but because it would be useful for comparison with other populations. The article does, however, raise various issues about depression in dementia.

Firstly, the symptoms overlap. The gold standard for depression used in this study was $I C D-10$. To be diagnosed as depressed an individual needs 4 items that include: loss of interest and enjoyment, increased fatigue, reduced concentration and attention, and disturbed sleep. Many clinicians may consider these symptoms to be part of AD.

Secondly, symptoms may not be reported. Patients may forget symptoms or find them difficult to articulate. Symptoms fluctuate. Patients may find it difficult to answer the BDI on a computer screen even with help from their carers.

Thirdly, the BDI was not designed as a diagnostic instrument for the elderly. Various somatic symptoms are included in the scale and these are common in non-depressed older people. Interestingly, the authors found that the somatic symptoms were more common in depressed than nondepressed patients, but they were not as useful as other symptoms in separating the 2 groups.

So, where does this leave us? Depression is common in dementia. It is unpleasant for patients and carers, and there is some evidence that it is treatable with antidepressants or behaviour therapy. ${ }^{1}$ The search is on for other scales that may prove useful for screening. The Geriatric Depression Scale was designed for depression in the elderly, and appears to be useful in patients with mild to moderate dementia. ${ }^{2}$ The Cornell Scale for Depression in Dementia uses information from caregivers and relies more on observable symptoms such as agitation, weight loss, and early morning wakening. Perhaps these scales (or others) will succeed where the BDI has failed.

Rob Butler, MBBS, MRCPsych Imperial College Middlesex, UK

1 Butler R. The diagnosis and management of depression in dementia. CPD Bulletin Old Age Psychiatry 1998;1:9-11.

2 Katz IR. Diagnosis and treatment of depression in patient with Alzheimer's disease and other dementias. J Clin Psychiatry 1998;59(suppl 9):38-44.
Source of funding: no external funding.

For correspondence:

Dr GE Berrios,

University of

Cambridge,

Department of

Psychiatry,

Addenbrooke's Hospital (Box 189), Hills Road,

Cambridge CB2 2QQ

UK. Fax +44 (0) 1223 336968 . 